\title{
The Impact of Income and Taxation in a Price-Tiered Cigarette Market: findings from the ITC Bangladesh Surveys
}

\author{
Iftekharul Huq, ${ }^{1}$ Nigar Nargis, ${ }^{2}$ Damba Lkhagvasuren, ${ }^{3}$ AKM Ghulam Hussain, ${ }^{4}$ \\ Geoffrey T Fong ${ }^{5,6}$
}

- Additional material is published online only. To view please visit the journal online (http://dx.doi.org/10.1136/ tobaccocontrol-2017-054053).

'Department of Economics, East West University, Dhaka, Bangladesh

${ }^{2}$ Economic and Health Policy Research Unit, American Cancer Society, Atlanta, Georgia, USA

${ }^{3}$ Department of Economics, Concordia University, Montreal, Quebec, Canada

${ }^{4}$ Department of Economics, University of Dhaka, Dhaka, Bangladesh

${ }^{5}$ Department of Psychology and School of Public Health and Health Systems, University of Waterloo, Waterloo, Ontario, Canada

${ }^{6}$ Ontario Institute for Cancer Research, Toronto, Ontario, Canada

Correspondence to Dr. Iftekharul Huq, Department of Economics, East West University, Dhaka 1212, Bangladesh; iftekharulhuq.12@gmail.com

Received 19 September 2017 Revised 24 February 2018 Accepted 21 March 2018 Published Online First 25 April 2018

\begin{abstract}
Background Taxing tobacco is among the most effective measures of tobacco control. However, in a tiered market structure where multiple tiers of taxes coexist, the anticipated impact of tobacco taxes on consumption is complex. This paper investigates changing smoking behaviour in lieu of changing prices and changing income. The objective of the paper is to evaluate the effectiveness of change in prices (through taxes) and change in income in a price-tiered cigarette market.
\end{abstract}

Method A panel dataset from the International Tobacco Control Bangladesh surveys is used for analysis. For preliminary analysis transition matrices are developed. Next, probit and multinomial logit regression models are used to identify the effects of changes in prices and changes in income along with other control variables.

Findings Transition matrices show significant movement of smokers across price tiers from one wave to another. Regression results show that higher income raises the probability to up-trade and decreases the probability to down-trade. Results also show that higher prices raises the probability to up-trade and reduces the probability to down-trade. Although not significant, there exists a negative relationship between the probability to down-trade and the probability to intend to quit.

Conclusion It is evident from the results that a pricetiered market provides smokers more opportunities to accommodate their smoking behaviour when faced with price and income change. Therefore, tiered structure of the tax system should be replaced with uniform taxes. Moreover, overall cigarette taxes need to be raised to an extent so that it off-sets any positive effects of income growth.

\section{INTRODUCTION}

Bangladesh is one of the largest tobacco-consuming countries in the world. In fact, it lies among the top 10 countries in the world with the high current smoking prevalence of $44.7 \%$ among men. ${ }^{1}$ Throughout the decade, sporadic measures have been undertaken by the Government of Bangladesh in an attempt to arrest the growth of tobacco consumption. These measures include taxing tobacco products, warning labels on cigarette packs in text form and smoking ban in public places. Despite these measures, the use of tobacco in the country continues unabated.

In Bangladesh, the government has segmented the market for cigarettes on the basis of prices, namely, low, medium, high and premium. Each tier is then taxed at a different rate. Typically, the lower tiered markets are taxed at lower rates than their higher counterparts. This type of tiered tax structure creates larger price differentials between high-priced and low-priced tobacco product, thus providing smokers with the opportunity to substitute to cheaper tobacco alternatives rather than to quit altogether, as tobacco taxes and prices rise and income levels decrease. ${ }^{2}$

In a dynamic setting, changes in prices of cigarettes are coupled with changes in income levels. A comprehensive analysis based on both price as well as income changes is called for to understand smokers' behaviour in a price-tiered market. When faced with changing income, smokers have different choices such as to continue to smoke, as usual, to quit, to change the number of cigarettes smoked per day or to switch to a different price tier of the market.

The objective of this paper is to analyse smoking behaviour in the face of income change along with price change when there exists a price-tiered market structure. Smoking behaviour is measured in the following two ways: (A) up-trade (move to a higher price tier); and (B) down-trade (move to a lower price tier). In a separate exercise, the paper also addresses to what extent smokers' ability to down-trade between price tiers leads to a reduction in the probability of quitting.

This paper adds to existing literature in two ways. The brand-switching behaviour of smokers in a price-tiered market as in Bangladesh is a relatively less studied area. The paper contributes to a better understanding of this behaviour in relation to the changing income pattern of smokers. Also, analysis with respect to change in income using a panel data has not been carried out before in Bangladesh.

The analysis uses the panel data collected across 3 years by International Tobacco Control (ITC) Bangladesh Surveys. The data were collected in 2009, 2010 and 2011-2012. The main advantage of analysing a panel data is that it gives the opportunity to track the behaviour of the same individual across time, thereby observing smoking trajectories such as switching among tiers. The additional appeal to the dataset is that it is relatively recent therefore revealing updated individual statistics.

The study of the brand-switching behaviour of smokers is not new in literature. However, the brand-switching literature is mixed in terms of the direction. Suranovic et al and Sutton et al showed brand-switching behaviour is intended to offset the 
increased cost of smoking from reduced smoking resulting from higher cigarette prices. ${ }^{3}{ }^{4}$ Furthermore, studies by Armitage et al, Bader et al, Guyatt et al, White et al, Li et al, Hasenfratz et $a l$ and Woodman et al concluded that smokers would substitute their preferred brands with lower price brands in order to maintain the total number of cigarettes consumed when facing higher cigarette taxes. ${ }^{5-11}$ In fact, financial motivation is the key factor behind economic compensation.

In a recent paper, Nargis et al found that the prevalence of exclusive cigarette smoking actually went up from 2009 to 2012 in Bangladesh despite a gradual yearly increase in tobacco taxation during the same period. ${ }^{12}$ In another paper, Nargis et al derived that insignificant increase in tobacco taxation failed to deliver expected results in Bangladesh as the likely negative effect of a modest price increase on inelastic cigarette demand was more than offset by a strong positive effect on income growth in Bangladesh. Even though tobacco products have been taxed in the past, it is considered to be insufficient. ${ }^{13}$

This paper accepts the results of Nargis et al that cigarette taxes are insignificant compared with large income growth. ${ }^{14}$ It thus addresses the issue of change in smoking behaviour in the face of income change. Second, it adds understanding of smoking behaviour by a more detailed analysis of the price-tiered market structure and observing the mobility of consumers across the different tiers in the face of income and price changes.

\section{DATA AND METHODOLOGY \\ Data}

The paper is based on data collected by the ITC surveys in Bangladesh. The ITC Bangladesh Project was launched in 2008 to evaluate the impact of tobacco control legislation in Bangladesh. The ITC Bangladesh Survey is a face-to-face survey conducted by the Bureau of Economic Research at the University of Dhaka, Bangladesh, in collaboration with the ITC Project team at the University of Waterloo in Canada. ${ }^{15}$

The paper uses data collected in the first three waves of the survey, conducted in 2009, 2010 and 2011-2012, respectively. The Wave 1 survey consisted of a nationally representative probability sample of smokers and non-smokers aged 15 years and older selected through a multistage cluster sampling design (sampling with probability proportional to population size at the levels of administrative units such as District, Upazila/Thana and Village/Ward). ${ }^{16}$ These respondents formed a cohort. They were contacted again to answer follow-up surveys in 2010 and 2011-2012. Between Waves 1 and 2, the overall retention rate is $92.4 \%$, whereas between Wave 2 and Wave 3, the rate is $90.2 \%{ }^{1718}$

The main objective of the study is to understand how smoker's behaviour changes over time. To do that, two datasets have been generated from the three waves of survey data. In this study, only male cigarette smokers are included from all waves, as the prevalence of smoking among female smokers is significantly lower (less than 2\%). In the first dataset generated, smokers from Wave 1 and Wave 2 are matched, and in the second one, smokers from Waves 2 and 3 are matched. These matched datasets provide scope to observe the change in smoker's behaviour over consecutive surveys. For the final analysis, both of these matched datasets are pooled. The total sample size thus becomes 4800 .

\section{Methodology}

For the purpose of data analysis, three binary outcome variables have been generated using the panel data. They are (A) up-trade or not, (B) down-trade or not and (C) intend to quit or not. In the case of the first binary variable, when an individual switches to a higher price tier compared with his previous wave's price tier, a value of 1 is assigned to the individual, otherwise 0 is assigned. Similarly, for the second binary variable, when an individual switches to a lower price tier compared with the individual's previous wave's price tier, 1 is assigned to him, otherwise 0 is assigned. For the third one, one represents smokers' intention to quit within the next 6 months and 0 represents otherwise.

On the explanatory side, there are three main explanatory variables: (A) change in price, (B) change in income and (C) change in addiction level. In data, prices have been reported by individuals. For robustness, in the probit, multinomial logit and hazard models, four different models with four different measures of changes in prices has been used. In the first model, change in reported prices are taken as an independent variable; however, such measurements create the possibility of endogeneity bias. In the second model, changes in taxes are used. Based on the reported prices, tax rates are assigned, and then changes are calculated and used as proxies of changes in prices. In the third model, changes in village-level average prices are also used to capture the price changes, and in the last model, changes in predicted prices are used as changes in prices.

In the dataset, the individuals reported a particular range within which their income lies as opposed to the exact income in figures. To observe the real income, income categories have been assigned with midpoint values of the respective income category. These midpoints are adjusted for inflation. For the purpose of the study, a new variable 'change in income' is generated by calculating the difference between the midpoints in consecutive surveys.

To capture the impact of preference, an additional variable is formed by observing how long after waking up in the morning the individual lights up the first cigarette. This indicates an individual's dependency on smoking. The quicker a person lights up, the more addicted he is. The more addicted he is, the higher will be the smoking preference. Two categories of smokers are formed for the analytical purpose. One as less addicted smokers who light up their first cigarette after $60 \mathrm{~min}$ and the other as more addicted smokers who light up within the first $60 \mathrm{~min}$ of waking up. A 'change in addiction level' variable is generated by observing the difference between two waves' addiction levels. A positive value represents an increase in preference towards smoking, and a negative value represents the opposite.

Finally, the impact of demographics on smoking behaviour has been captured through the following variables: education, age and residence (rural or urban). Individuals are assigned to three broad education category: illiterate, less educated (1-8 years of schooling) and more educated ( 9 and more years of schooling).

\section{DESCRIPTIVE STATISTICS}

Bangladesh uses a tiered cigarette tax structure that imposes different ad valorem tax rates known as excise tax (supplementary duty) based on retail price slabs. In addition, there is a value added tax of $15 \%$ of the retail price. In Bangladesh, all different brands of cigarettes are divided into four tiers based on their prices; low, medium, high and premium. Between 2009 and 2012, there was a steady increase in cigarette prices for each of the four retail price slabs, but increases in the SD (supplementary

\footnotetext{
${ }^{\mathrm{i}}$ Positive values indicate an increase in real income whereas negative values signify a decrease in real income. This 'Change in income' variable is then divided by 1000 to identify the effect of per BDT 1000 change on different tobacco behaviour parameters.
} 
Table 1 Bangladesh cigarette tax structure 2009-2012*

\begin{tabular}{llll}
\hline $\begin{array}{l}\text { Year of } \\
\text { observation }\end{array}$ & Price tier & BDT/pack of 10 & $\begin{array}{l}\text { Excise tax } \\
\text { (\% of retail price) }\end{array}$ \\
\hline Wave 1 (2009) & Low & $7.25-8.75$ & 32 \\
& Medium & $16.25-17.25$ & 52 \\
& High & $23.25-29.25$ & 55 \\
& Premium & $46.25+$ & 57 \\
Wave 2 (2010) & Low & $8.40-9.15$ & 33 \\
& Medium & $18.40-19.00$ & 53 \\
& High & $27.00-32.00$ & 56 \\
& Premium & $52.00+$ & 58 \\
Wave 3 (2011- & Low & $11.00-11.30$ & 36 \\
2012) & Medium & $22.50-23.00$ & 55 \\
& High & $32.00-36.00$ & 58 \\
& Premium & $60.00+$ & 60 \\
\hline
\end{tabular}

*National Board of Revenue, Government of Bangladesh.

duty) for each price slab were marginal (see table 1). It should also be noted that because the range of price bands are not continuous, there are significant price gaps between bands in different price categories. This gap provides incentives for tax avoidance and evasion among manufacturers (eg, by positioning brands in the gaps between price slabs, tobacco companies can pay lower taxes because the provision for penalties for selling beyond the maximum price in the slab is not currently being enforced).

The table above illustrates the tax on cigarette packs across the three waves. Tax rates imposed on the lower tiers are significantly lower than the higher ones. Excise taxes on cigarettes account for just over one-half of retail prices on average. This falls well below the level in countries with strong tobacco control policies where excise taxes typically account for more than $70 \%$ of retail price. ${ }^{19}$

Table 2 shows the distribution of smokers across different price tiers over the 3 years. It is apparent that smokers' presence in different price tiers changed drastically over the years. Smokers belonging to lowest price tier increased from 29.64\% to $42.82 \%$ in just 2 years. In contrast, medium price tier experienced a significant reduction in terms of smokers' proportion from $46.15 \%$ to $31.30 \%$. However, the top two price tiers did not witness any major shifts over the years. It is this evidence that feeds interest to the question of smokers' mobility across price tiers over the years.

Observations from table 2 prompted to give more structure to changing patterns in smokers' mobility across price tiers. To that direction, transition matrices were generated to identify mobility within different price tiers for cigarette smokers.

\begin{tabular}{lccc}
\hline $\begin{array}{l}\text { Table } 2 \\
\text { waves }\end{array}$ & \multicolumn{2}{l}{ Distribution of smokers in different price tiers in the three } \\
\hline \multirow{4}{*}{$\begin{array}{l}\text { Cigarette } \\
\text { price tier }\end{array}$} & \multicolumn{3}{l}{ Percentages of smokers in each price tier } \\
\cline { 2 - 4 } & Wave 1 & Wave 2 & Wave 3 \\
\hline Low & 29.64 & $(\mathbf{2 0 1 0 )}$ & $(\mathbf{2 0 1 1 - 2 0 1 2 )}$ \\
\hline Medium & 46.15 & 39.06 & 42.82 \\
\hline High & 16.51 & 33.50 & 31.30 \\
\hline Premium & 7.70 & 19.88 & 17.11 \\
\hline All & 100 & 7.56 & 8.77 \\
\hline
\end{tabular}

Table 3 Transition matrices: movement of smokers from one wave to the next

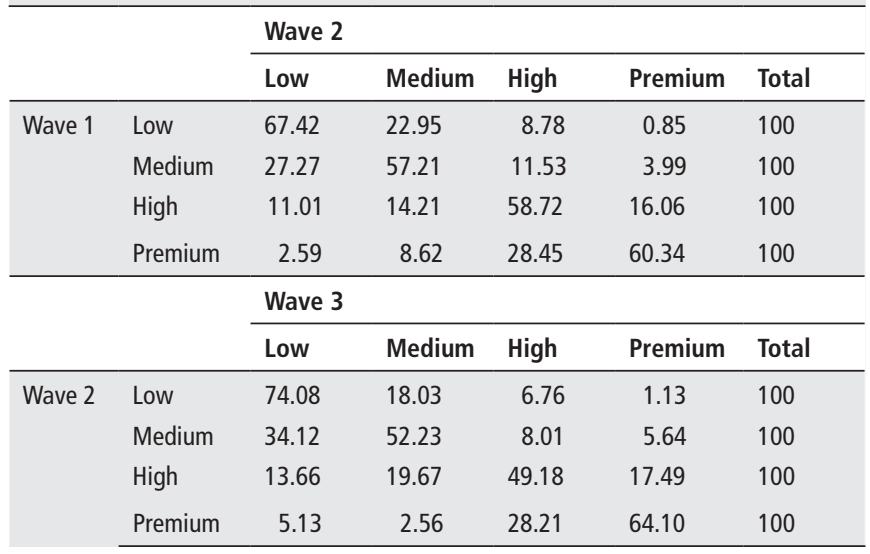

Several interesting observations come out of the transition matrices presented in table 3. First of all, compared with all the price tiers, smokers belonging to the 'low' category were least mobile. From the first to the second wave, $67.42 \%$ of the lowest price tier smokers remained in the same category (first transition matrix) compared with $57 \%, 59 \%$ and $60 \%$ in the higher categories. From the second to the third wave, $74.08 \%$ of the lowest price tier smokers remained immobile (second transition matrix).

Second, the rate of mobility is much higher among 'medium' and 'high' category. Among those who migrated, the tendency to down-trade is much higher than the tendency to up-trade. For example, $27.27 \%$ of smokers who smoked the medium type cigarettes in Wave 1 switched to the low type in Wave 2. In contrast, only $11.53 \%$ up-traded to the high and $3.99 \%$ to premium categories. Similar observations hold true in the second transition matrix as well. Thus, in the two middle price tiers, it seems that the tendency to down-trade dominates over the tendency to up-trade. This might be in response to decreasing income that encouraged switching to lower priced brands instead of quitting. Finally, looking at the two extreme price tiers, it is apparent in both transition matrices that the tendency for premium smokers to down-trade is higher than low priced smokers to up-trade.

In the dataset, individuals were required to report their income category, as opposed to actual income. Despite that, large variations in income can be found when generating transition matrices for income. Table 4 presents such transition matrices.

Above transition matrices presented in table 4 clearly show that income change had been a common phenomenon in

Table 4 Transition matrices: movement of smokers across different income groups from one wave to the next

\begin{tabular}{llllll}
\hline & & \multicolumn{3}{l}{ Wave 2} & \\
\cline { 3 - 5 } Wave 1 & Low & 77.38 & 18.05 & 4.56 & 100 \\
& Medium & 38.80 & 46.40 & 14.80 & 100 \\
& High & 15.85 & 37.20 & 46.95 & 100 \\
\hline \multirow{5}{*}{ Wave 2 } & Low & 67.13 & 29.18 & 3.70 & 100 \\
\cline { 3 - 5 } & & Low 3 & Medium & High & Total \\
& Medium & 25.49 & 54.67 & 19.84 & 100 \\
& High & 7.19 & 40.72 & 52.10 & 100 \\
\cline { 2 - 5 } & & & &
\end{tabular}


Table 5 Probit regression results: effects of changes in prices, income levels and addiction levels on probability to up-trade or not

\begin{tabular}{|c|c|c|c|c|}
\hline \multicolumn{5}{|c|}{ Dependent variable: probability to up-trade } \\
\hline \multirow[b]{2}{*}{ Variables } & \multicolumn{4}{|l|}{ Marginal effects } \\
\hline & Model 1 & Model 2 & Model 3 & Model 4 \\
\hline$\Delta$ in actual self-reported price & $0.012 *(0.002)$ & - & - & - \\
\hline$\Delta$ in actual tax & - & $0.017^{*}(0.001)$ & - & - \\
\hline$\Delta$ in village-level mean price & - & - & $0.007^{*}(0.003)$ & - \\
\hline$\Delta$ in predicted price & - & - & - & $0.010 *(0.002)$ \\
\hline$\Delta$ in income & $0.003(0.002)$ & $0.001(0.002)$ & $0.006^{*}(0.002)$ & $0.002(0.002)$ \\
\hline$\Delta$ in addiction & $0.019(0.018)$ & $0.008(0.017)$ & $0.002(0.019)$ & $0.017(0.019)$ \\
\hline
\end{tabular}

SEs are given in parentheses.

*At the $1 \%$ level.

tIndicates at the $10 \%$ level.

¥Indicates significance at the $5 \%$ level.

Bangladesh. A high proportion of smokers experienced a change in income category in just 1 year. Some of their income increased, while some faced reduced income as well. This change in income can have a consequential effect on smokers' behaviour towards smoking. Such change in income adds strength to the rationale of the study where the author wishes to investigate the change in smoking behaviour in the face of change in income.

In the summary statistics online supplementary appendix tables A1, A2, it is revealed that on average higher price tier cigarette smokers consume marginally fewer cigarettes per day and experience higher income growth. Mean values of these relatively more expensive cigarette smokers also show that they are more educated, younger, more likely to live in urban areas and also relatively less addicted to smoking. These results signify few interesting differences within smokers of different price tiers.

Online supplementary appendix table A3 illustrates some of the characteristics of up-traders and down-traders. While comparing mean values, it is revealed that up-traders consume fewer cigarettes per day and enjoy higher income growth as expected. Moreover, up-traders are more educated and living in urban areas. Down-traders show higher addiction towards smoking compared with up-traders. This implies that downtraders can be quantity loyal. Therefore, they choose to downtrade and maintain their quantity of cigarettes that satisfy their addiction level. These results help us identify few demographic differences between up-traders and down-traders.

\section{EMPIRICAL ANALYSIS}

In this paper, data analysis is carried out in three parts. First, a set of regression equations are formed to illustrate how up-trade and down-trade are affected by changes in prices, income levels and addiction levels.

In equation 1, the dependent variable is up-trade or not between two waves. In a similar way, regression equation 2 looks at down-trade or not. Both regression equations consist of identical independent variables that are change in price for which four different measures are used, change in income, change in addiction level along with demographic variables such as education groups, age, and residence (all are represented by $X)$ are used as independent variables in regressions 1 and 2 .

$$
\begin{aligned}
& \text { Prob }(\text { up }- \text { trade })=\alpha_{1}+\alpha_{2} \Delta \text { in price }+\alpha_{3} \Delta \text { in income }+ \\
& \text { Prob }(\text { down }- \text { trade })=\alpha_{4} \Delta \text { in gddiction }+\alpha_{1} X+\beta_{2} \Delta \text { in price }+\beta_{3} \Delta \text { in income }+ \\
& \beta_{4} \Delta \text { in addiction }+\beta_{5} X+\tau
\end{aligned}
$$

Table 5 gives the results of the first probit regression in terms of marginal effects. Findings from regression one reveal that higher prices are associated with smokers moving to higher price tiers. Such results are consistent across all four measures of price changes. Given the dataset, there were no appropriate instruments available in order to identify the effect of exogenous price shock. This remains a limitation of the analysis. Results also show that positive income change increases the likelihood for smokers to up-trade rather than remaining on the same price-tier or down-trading. An increase of BDT 1000 increases the probability to up-trade by $0.6 \%$. This is evidenced in the third measure of change in price (change in village-level average price). Change in addiction holds no significant effect on the probability to up-trade.

Table 6 Probit regression results: effects of changes in prices, income levels and addiction levels on probability to down-trade or not

\begin{tabular}{lllll}
\hline \multicolumn{2}{l}{ Dependent variable: probability to down-trade } & & & \\
\hline & \multicolumn{1}{l}{ Marginal effects } & Model 3 & Model 4 \\
\cline { 2 - 5 } Variables & Model 1 & Model 2 & - & - \\
\hline$\Delta$ in actual self-reported price & $-0.023^{*}(0.001)$ & - & - & - \\
\hline in actual tax & - & $-0.033^{*}(0.002)$ & $-0.013^{*}(0.003)$ & - \\
$\Delta$ in village-level mean price & - & - & - & $-0.014^{*}(0.003)$ \\
$\Delta$ in predicted price & - & - & $-0.007^{*}(0.003)$ & $-0.000(0.003)$ \\
$\Delta$ in income & $0.001(0.002)$ & $0.002(0.003)$ & $-0.041 \neq(0.024)$ & $-0.062 \dagger(0.025)$ \\
\hline in addiction & $-0.049 \dagger(0.019)$ & $-0.056 \dagger(0.023)$ & &
\end{tabular}

SEs are given in parentheses.

*At the $1 \%$ level.

tIndicates significance at the $5 \%$ level.

fIndicates significance at the $10 \%$ level. 
Table 7 Multinomial logit regression results

\begin{tabular}{|c|c|c|c|c|c|c|c|c|}
\hline \multirow[b]{3}{*}{ Variables } & \multicolumn{8}{|c|}{ Marginal effects } \\
\hline & \multicolumn{4}{|l|}{ Up-trade } & \multicolumn{4}{|c|}{ Down-trade } \\
\hline & Model 1 & Model 2 & Model 3 & Model 4 & Model1 & Model 2 & Model 3 & Model 4 \\
\hline $\begin{array}{l}\Delta \text { in actual self- } \\
\text { reported price }\end{array}$ & $\begin{array}{l}0.014^{*} \\
(0.002)\end{array}$ & - & - & - & $\begin{array}{l}-0.024^{*} \\
(0.002)\end{array}$ & - & - & - \\
\hline$\Delta$ in actual tax & - & $\begin{array}{l}0.017^{*} \\
(0.001)\end{array}$ & - & - & - & $\begin{array}{l}-0.029^{*} \\
(0.002)\end{array}$ & - & - \\
\hline $\begin{array}{l}\Delta \text { in village-level } \\
\text { mean price }\end{array}$ & - & - & $\begin{array}{l}0.006 t \\
(0.003)\end{array}$ & - & - & - & $\begin{array}{l}-0.008^{*} \\
(0.003)\end{array}$ & - \\
\hline $\begin{array}{l}\Delta \text { in predicted } \\
\text { price }\end{array}$ & - & - & - & $\begin{array}{l}0.010^{*} \\
(0.002)\end{array}$ & - & - & - & $\begin{array}{l}-0.008^{*} \\
(0.002)\end{array}$ \\
\hline$\Delta$ in income & $\begin{array}{l}0.003 \ddagger \\
(0.002)\end{array}$ & $\begin{array}{l}0.002 \\
(0.002)\end{array}$ & $\begin{array}{l}0.007^{*} \\
(0.002)\end{array}$ & $\begin{array}{l}0.003 \\
(0.002)\end{array}$ & $\begin{array}{l}-0.001 \\
(0.002)\end{array}$ & $\begin{array}{l}-0.000 \\
(0.002)\end{array}$ & $\begin{array}{l}-0.005 t \\
(0.002)\end{array}$ & $\begin{array}{l}-0.002 \\
(0.002)\end{array}$ \\
\hline$\Delta$ in addiction & $\begin{array}{l}0.006 \\
(0.016)\end{array}$ & $\begin{array}{l}0.000 \\
(0.016)\end{array}$ & $\begin{array}{l}-0.002 \\
(0.019)\end{array}$ & $\begin{array}{l}0.010 \\
(0.019)\end{array}$ & $\begin{array}{l}-0.023 \ddagger \\
(0.013)\end{array}$ & $\begin{array}{l}-0.025 \ddagger \\
(0.014)\end{array}$ & $\begin{array}{l}-0.028 \\
(0.018)\end{array}$ & $\begin{array}{l}-0.037 \ddagger \\
(0.019)\end{array}$ \\
\hline
\end{tabular}

Unchanged price tier is the base category. SEs are given in parentheses.

${ }^{*}$ At the $1 \%$ level.

tAt the $5 \%$ level.

flndicates significance at the $10 \%$ level.

Findings from the second probit regression displayed in table 6 reveal that an increase in price reduces the probability to down-trade. As explained earlier, lack of an appropriate instrument in the dataset remains a constraint in appropriately measuring exogenous price shock. Results also show that higher income reduces the probability to down-trade compared with other alternatives. Results from the marginal effects reveal that an increase of BDT 1000 reduces the likelihood to down-trade by $0.7 \%$. In addition, more addicted smokers are less likely to down-trade. Full results of the probit regression models are presented in online supplementary appendix tables A4, A5.

To strengthen this exercise, a multinomial logit regression model is also used. The dependent variable is formulated such a way so that 0 is assigned to smokers whose price tier remain unchanged between two waves, whereas 1 is assigned to smokers who up-trades and 2 is assigned to smokers who down-trades. In the multilogit regression model, 0 is chosen to be the base category.

$$
\begin{aligned}
\Delta \text { in price }- \text { tier }= & \gamma_{1}+\gamma_{2} \Delta \text { in price }+\gamma_{3} \Delta \text { in income }+ \\
& \gamma_{4} \Delta \text { in addiction }+\gamma_{5} X+\eta
\end{aligned}
$$

Findings from multinomial logit regression model are presented in table 7 .

This exercise reveals similar results to the previous one. Again we find an increase in price increases the probability to up-trade and reduces the probability to down-trade. Increase in income increases the probability to up-trade and reduces the probability to down-trade. The level of addiction holds no significant impact on the probability to up-trade; however, it reduces the probability to down-trade. Full results of the multilogit regression models are presented in online supplementary appendix table A6.

In the second part of the analysis, this paper investigates to what extent smokers' ability to down-trade between price tiers leads to a reduction in the probability of quitting due to the current system of price tiers. Such an exercise is important because the existence of an opportunity to down-trade may be a key factor encouraging smokers to continue smoking. This holds significant policy implications from public health perspectives. A probit regression is introduced where the dependent variable represents the probability to intend to quit or not. For the binary dependent variable, 1 represents smokers' intention to quit within the next 6 months and 0 represents otherwise. Along with all the independent variables from the previous models, a binary variable is added where 1 denotes down-trading and 0 otherwise.

$$
\begin{gathered}
\text { Prob }(\text { intend to quit })=\delta_{1}+\delta_{2} \text { Down }- \text { trade }+\delta_{3} \Delta \text { in price }+ \\
\delta_{4} \Delta \text { in income }+\delta_{5} \Delta \text { in addiction }+\delta_{6} X+\mu
\end{gathered}
$$

Results from table 8 show that the probability is lower among those who down-trade. However, the results are not statistically significant. Full results of the probit regression models are presented in online supplementary appendix table A7.

In the last part of the analysis, this paper introduces the Cox regression models to analyse the association between smokers' change in behaviour and changes in price, income and addiction level. This exercise enhances the robustness of the previous findings. For the following analysis, price, income and addiction variables are modified. Rather than looking at change in price, income and addiction level (as is the case with probit and multinomial logit models) in this model, the consideration is whether an individual faces an increase in price, income and addiction level or not. For each of these variables, a value of 1 represents an increase of the respective variable and 0 represents otherwise. In the corresponding Cox regression models age, residence (rural/urban) and education levels are also included as covariates. The results are presented below.

Overall, the results are consistent to the previous results from the probit and multinomial logit regression models. In the top section of table 9, up-trading is denoted as the event in measuring the HRs. It is observed that higher prices increase the likelihood to up-trade. Moreover, results also illustrated that an increase in income is associated with 63\% (model 3) increase in HRs, meaning that higher income positively affected up-trading.

In the middle section of table 9, down-trading is assigned as the event and results revealed that higher prices reduce the likelihood to down-trade. Although it is observed that an increase in income decreases the probability to down-trade, the results are not statistically significant.

In the last section of table 9, the incidence of quitting is denoted as the event. Results show that down-trading is negatively associated with quitting; however, the results are not statistically significant. Similar results are found in table 8. Full 
Table 8 Probit regression results: effects of down-trading, changes in prices, income levels and addiction levels on probability to intend to quit or not

\begin{tabular}{|c|c|c|c|c|}
\hline \multicolumn{5}{|c|}{ Dependent variable: probability to intend to quit } \\
\hline \multirow[b]{2}{*}{ Variables } & \multicolumn{4}{|l|}{ Marginal effects } \\
\hline & Model 1 & Model 2 & Model 3 & Model 4 \\
\hline $\begin{array}{l}\text { Down-trade or not } \\
\text { (1: down-trade; } 0 \text { : otherwise) }\end{array}$ & $-0.012(0.042)$ & $-0.007(0.043)$ & $-0.015(0.033)$ & $-0.015(0.034)$ \\
\hline$\Delta$ in actual self-reported price & $0.000(0.001)$ & - & - & - \\
\hline$\Delta$ in actual tax & - & $0.001(0.002)$ & - & - \\
\hline$\Delta$ in village-level mean price & - & - & $0.006(0.004)$ & - \\
\hline$\Delta$ in predicted price & - & - & - & $0.003(0.003)$ \\
\hline$\Delta$ in income & $0.003(0.002)$ & $0.003(0.002)$ & $0.003(0.002)$ & $0.002(0.003)$ \\
\hline$\Delta$ in addiction & $0.074^{*}(0.022)$ & $0.073^{*}(0.022)$ & $0.077^{*}(0.021)$ & $0.077^{*}(0.022)$ \\
\hline
\end{tabular}

SEs are given in parentheses.

*At the $1 \%$ level.

tIndicates significance at the $10 \%$ level.

fIndicates significance at the $5 \%$ level.

results of the Cox regression models are presented in online supplementary appendix table A8.

\section{DISCUSSION AND CONCLUDING REMARKS}

In this paper, analysis is carried out using the ITC survey of Bangladesh to understand smokers' behaviour in a tiered cigarette market structure. In Bangladesh, there are four distinct price tiers that exist in the cigarette market. It is observed from the distribution of smokers across price tiers that percentages of smokers in the lowest price tier are rising over the years, and percentages of smokers in the middle price-tier are shrinking. Smokers' prevalence in the top two price tiers remain relatively stable over the years.

This paper presents transition matrices to observe smokers' movements across different price-tiers from one wave to the next. It is observed in the transition matrices that significant percentages of smokers move across price tiers over the years. Both transition matrices illustrated that down-trading is more

Table 9 Cox regression results: HRs

\begin{tabular}{|c|c|c|c|c|}
\hline \multirow[b]{2}{*}{ Up-trade } & \multicolumn{4}{|l|}{ HRs $(95 \% \mathrm{Cl})$} \\
\hline & Model $1(n=1507)$ & Model $2(n=1500)$ & Model $3(n=1651)$ & Model $4(n=1518)$ \\
\hline Actual self-reported price & $5.778 *$ (3.678 to 9.077 ) & - & - & - \\
\hline Village-level mean price & - & - & $1.483+$ (1.096 to 2.006$)$ & - \\
\hline Predicted price & - & - & - & $1.932 *$ (1.347 to 2.772$)$ \\
\hline Income & $1.511^{*}$ (1.129 to 2.022$)$ & $1.542^{*}$ (1.151 to 2.064$)$ & $1.631^{*}$ (1.225 to 2.172$)$ & $1.380+$ ( 1.021 to 1.866$)$ \\
\hline Addiction & \multicolumn{4}{|l|}{ HRs $(95 \% \mathrm{Cl})$} \\
\hline Down-trade & Model $1(n=1507)$ & Model $2(n=1500)$ & Model $3(n=1651)$ & Model $4(n=1518)$ \\
\hline Actual self-reported price & $0.080 *(0.048$ to 0.132$)$ & - & - & - \\
\hline Actual tax & - & $0.056^{*}(0.037$ to 0.083$)$ & - & - \\
\hline Village-level mean price & - & - & $0.754 \dagger(0.574$ to 0.992$)$ & - \\
\hline Quit & Model $1(n=709)$ & Model $2(n=706)$ & Model $3(n=751)$ & Model $4(n=713)$ \\
\hline Down-trade & 0.977 (0.487 to 1.960$)$ & 0.995 (0.446 to 2.219$)$ & 0.901 (0.545 to 1.488$)$ & $0.884(0.532-1.468)$ \\
\hline Actual self-reported price & 1.115 (0.589 to 2.111$)$ & - & - & - \\
\hline Actual tax & - & 1.141 (0.515 to 2.529$)$ & - & - \\
\hline Village-level mean price & - & - & $1.266(0.812$ to 1.974$)$ & - \\
\hline Predicted price & - & - & - & 0.959 (0.592 to 1.552$)$ \\
\hline Income & 1.422 (0.924 to 2.188$)$ & 1.428 (0.930 to 2.195$)$ & $1.422(0.927$ to 2.181$)$ & 1.427 (0.911 to 2.235$)$ \\
\hline Addiction & $1.841+(1.134$ to 2.988$)$ & $1.829+$ (1.127 to 2.969$)$ & $1.971^{*}(1.222$ to 3.178$)$ & $1.833+$ (1.121 to 2.998$)$ \\
\hline
\end{tabular}

${ }^{*}$ At the $1 \%$ level.

tIndicates significance at the $5 \%$ level.

fIndicates significance at the $10 \%$ level. 
prevalent than up-trading. Another set of transition matrices are then generated to observe smokers' movement across different income groups over the years. These matrices reveal significant levels of mobility of smokers across income groups.

Based on the observations from data, probit models has been designed to explain the change in smokers' behaviour based on two main explanatory variables, namely, change in price and change in income. For robustness, four different measures of changes in prices are used. Results show that an increase in price increases the likelihood to up-trade and reduces the likelihood to down-trade. Such results are consistent across all four measures of price changes. Given the dataset, there were no appropriate instruments available in order to identify the effect of exogenous price shock. This remains a limitation of the analysis.

Results also show that positive income change increases the likelihood for smokers to up-trade, and it reduces the likelihood for smokers to down-trade. Thus, it is shown that changes in income levels has significant impact on smoking behaviour in terms of changing price tiers. A multinomial logit regression is also carried out in this paper. Findings from the multinomial logit regression are consistent with the findings from the probit regression models.

An exercise to identify how the intention to quit gets affected with the probability to down-trade is also carried out in this paper. Even though the results are not statistically significant, the paper finds a negative relationship indicating that when there exists an opportunity to down-trade, smokers' intention to quit smoking goes down.

Empirical results of this paper is further strengthened by including Cox regression models to measure HRs. Findings from Cox regression models are consistent with the previous probit and multinomial logit regression results. Such outcomes enhance the overall acceptability of the models presented in this paper.

The results from this paper clearly demonstrate that in a country such as Bangladesh where movements across income groups are very common even in a span of 1 year, the presence of differently taxed price-tiers has a significant impact on smoking behaviour. Multiple price tiers give smokers ample room of flexibility in the face of any income shocks. In order to prevent smokers from switching tiers (as opposed to quitting), the level of tax should be higher on the lower tiered brands. Proponents of the current structure (where the lower price tiers are taxed at a lower slab) argue that reversing the system would be highly regressive in nature. After all, data reveals that the lower income groups are the main consumers of cigarettes in the lowest price tiers. Raising the tax rates in these groups would in effect tax the poor. However, it must be kept in mind that the objective of taxing tobacco is not income equality, rather it is to prevent consumption of a social bad. Thus, when accounting for the health benefits that arise from quitting and reduced expenditures that the poor will enjoy from quitting, a harsh but effective tax on the lower tiers of the market may well prove to be socially desirable. Furthermore, tax gaps need to be reduced between different cigarette price bands. Results from this paper highlight the importance of implementing a uniform specific tax system that will maximise price increases and decreases in consumption.

Finally, any successful policy structure to curb a social bad such as cigarettes should include pecuniary as well as non-pecuniary incentives. Examples of non-pecuniary policies can be to increase awareness of harmful effects of smoking, introduce graphic warnings on cigarette packs, impose a public ban on smoking, create role models/personalities that publicly debauch smoking and so on. To reduce consumption as well as increase the number of successful quitters, a more holistic approach to policy design needs to be implemented.

\section{What this paper adds}

- This paper is the first analysis in identifying the pattern of brand switching in the context of Bangladesh.

- The paper then adds explanation to the pattern through income change and price change.

- The results emphasise the importance of implementing a uniform tax system rather than having a tiered structure in order to have more effective policies.

Correction notice This article has been corrected since it was published Online First. Additional details of support were added to the Funding section.

Acknowledgements We would like to thank the data management staff at the ITC Data Management Center of the University of Waterloo who processed the data for use and also the team of interviewers at the University of Dhaka who collected data through face-to-face interviews in Bangladesh.

Contributors $\mathrm{IH}$ is the lead author of the paper. NN and DL contributed in developing the research idea. AKMGH contributed in the econometric analysis. GTF is the chief investigator of the ITC Project and a co-author of the paper.

Funding The ITC Bangladesh Survey was supported by the International Development Research Centre (IDRC Grant 104831-002) and Canadian Institutes of Health Research (MOP 79551 and MOP 115016), and the US National Cancer Institute (P01 CA138389). Additional support was provided to GTF from a Senior Investigator Award from the Ontario Institute for Cancer Research and a Prevention Scientist Award from the Canadian Cancer Society Research Institute. Additional support in preparing this paper was provided to University of Waterloo by the Canadian Institutes of Health Research (FDN-148477).

Disclaimer The authors alone are responsible for the views expressed in this article, and they do not necessarily represent the views, decisions, or policies of the institutions with which they are affiliated.

Competing interests None declared.

Patient consent Obtained.

Ethics approval Bangladesh Medical Research Council and the Office of Research Ethics, University of Waterloo, Canada.

Provenance and peer review Not commissioned; externally peer reviewed.

(c) Article author(s) (or their employer(s) unless otherwise stated in the text of the article) 2019. All rights reserved. No commercial use is permitted unless otherwise expressly granted.

\section{REFERENCES}

1 World Health Organization. Global adult tobacco survey: Bangladesh report. 2009 http://www.who.int/tobacco/surveillance/global_adult_tobacco_survey_bangladesh_ report_2009.pdf (accessed Dec 2016).

2 Chaloupka FJ, Kostova D, Shang C. Cigarette excise tax structure and cigarette prices: evidence from the global adult tobacco survey and the U.S. national adult tobacco survey. Nicotine Tob Res 2014;16(Suppl 1):S3-S9.

3 Suranovic SM, Goldfarb RS, Leonard TC. An economic theory of cigarette addiction. Journal of Health Economics 1999;18:1-29.

4 Sutton SR, Feyerabend C, Cole PV, et al. Adjustment of smokers to dilution of tobacco smoke by ventilated cigarette holders. Clin Pharmacol Ther 1978;24:395-405.

5 Armitage AK, Alexander J, Hopkins R, et al. Evaluation of a low to middle tar/ medium nicotine cigarette designed to maintain nicotine delivery to the smoker. Psychopharmacology 1988;96:447-53.

6 Bader P, Boisclair D, Ferrence R. Effects of tobacco taxation and pricing on smoking behavior in high risk populations: a knowledge synthesis. Int J Environ Res Public Health 2011:8:4118-39.

7 Guyatt AR, Kirkham AJ, Mariner DC, et al. Long-term effects of switching to cigarettes with lower tar and nicotine yields. Psychopharmacology 1989;99:80-6.

8 White JS, Li J, Hu TW, et al. The effect of cigarette prices on brand-switching in China: a longitudinal analysis of data from the ITC China Survey. Tob Control 2014;23(Suppl 1):i54-60

9 Li J, White JS, Hu TW, et al. The heterogeneous effects of cigarette prices on brand choice in China: implications for tobacco control policy. Tob Control 2015;24(Suppl 3):iii25-32.

10 Hasenfratz M, Baldinger B, Bättig K. Nicotine or tar titration in cigarette smoking behavior? Psychopharmacology 1993;112:253-8. 
11 Woodman G, Newman SP, Pavia D, et al. Response and acclimatisation of symptomless smokers on changing to a low tar, low nicotine cigarette. Thorax 1987;42:336-41.

12 Nargis N, Thompson ME, Fong GT, et al. Prevalence and patterns of tobacco use in Bangladesh from 2009 to 2012: evidence from International Tobacco Control (ITC) Study. PLoS One 2015;10:e0141135.

13 Nargis N, Stoklosa M, Drope J, et al. The trend in affordability of tobacco products in Bangladesh 2009-2015: Evidence from ITC Bangladesh Surveys. Waterloo: University of Waterloo, 2016.

14 Nargis N, Ruthbah UH, Hussain AK, et al. The price sensitivity of cigarette consumption in Bangladesh: evidence from the International Tobacco Control (ITC) Bangladesh Wave 1 (2009) and wave 2 (2010) surveys. Tob Control 2014;23(Suppl 1):i39-i47.
15 ITC Bangladesh technical report wave 1. 2010 http://www.itcproject.org/files/ bd1trfinalapr21.pdf (accessed Oct 2016).

16 ITC Bangladesh technical report wave 2. $2016 \mathrm{http}: / /$ www.itcproject.org/files/BD2-TRFinal_June62016.pdf (accessed Oct 2016).

17 ITC Bangladesh Technical Report Wave 3. 2015 http://www.itcproject.org/files/ITC Bangladesh Wave 3 Technical_Report-FINAL-May2016.pdf (accessed October 2016)

18 ITC Project. Tobacco price and taxation policies in Bangladesh: evidence of effectiveness and implications for action. Waterloo : University of Waterloo, 2014.

19 World Health Organization. WHO technical manual on tobacco tax administration. World Health Organization, 2010. 